\title{
Facile Synthesis of Graphite-Reduced Graphite Oxide Core-Sheath Fiber via Direct Exfoliation of Carbon Fiber for Supercapacitor Application
}

\author{
Qinghua Ji, ${ }^{\dagger \dagger} \mathrm{Xu} \mathrm{Zhao}^{\dagger}$ Huijuan $\mathrm{Liu}^{\dagger}{ }^{\dagger}$ Lin Guo, ${ }^{\S}$ and Jiuhui $\mathrm{Qu}^{\dagger}{ }^{\dagger} *$ \\ ${ }^{\dagger}$ Key Laboratory of Aquatic Science and Technology, Research Center for Eco-Environmental Sciences, Chinese Academy of \\ Sciences, Beijing 100085, China \\ ${ }^{\ddagger}$ University of Chinese Academy of Sciences, Beijing 100049, China \\ ${ }^{\S}$ School of Chemistry and Environment, Beihang University, Beijing 100191, China
}

Supporting Information

ABSTRACT: A graphite-reduced graphite oxide (rGO) core-sheath structured fiber was synthesized through chemical exfoliation of graphitic carbon fiber. The graphitic carbon fiber was oxidized to form a graphitegraphite oxide core-sheath fiber and followed by thermal exfoliation to form a graphite-rGO core-sheath fiber. The core-sheath fiber with a three-dimensionally (3D) structured rGO sheath possesses a high surface area and pore size around $5.5 \mathrm{~nm}$. A two-electrode supercapacitor constructed with this core-sheath fiber-based paper exhibited a high specific capacitance $\left(140 \mathrm{~F} \mathrm{~g}^{-1}\right.$ at a current density of $\left.1 \mathrm{~A} \mathrm{~g}^{-1}\right)$, high power density of $45 \mathrm{~kW} / \mathrm{kg}$, and good cycling stability (10\% capacity loss after 3000 cycles). The surface area normalized capacitance reached as high as $59.4 \mu \mathrm{F} \mathrm{cm}^{-2}$, indicating the effective use of surface area. The low equivalent series resistance value of $0.45 \mathrm{ohm}$ in the Nyquist plot indicates an extremely small resistance between the graphite core and rGO sheet sheath. The hierarchical three-dimensional structure enables one to maximize the advantages of both graphite and rGO sheets. The 3D-structured rGO sheets sheath with regular pore structure is favorable for ion diffusion due to its interconnected porous system, while the graphite core provides an electron transport pathway with high conductivity.

KEYWORDS: graphene, carbon fiber, core-sheath structure, supercapacitor

\section{INTRODUCTION}

Graphene, with unique properties such as high surface area, electrical and thermal conductivity, is a promising candidate for applications in nanoelectronic devices, energy storage, and other applications. ${ }^{1-4}$ Currently, graphite oxide (GO), available in bulk quantities at low cost, ${ }^{5}$ has been used as a building block for production of many graphene-based materials. ${ }^{6-12}$ However, these graphene-based materials show poor electrical conductivity due to severe structural defects in the graphene sheets caused by chemical oxidation and reduction. Moreover, the specific surface areas are usually decreased during the processes of synthesizing graphene-based materials owing to the $\pi-\pi$ stacking between graphene sheets. The construction of a three-dimensional (3D) porous framework can partially overcome the problem of restacking and aggregation. A variety of methods have been developed for fabricating such materials from GO, including self-assembly, template-guided growth, and organic sol-gel reaction. ${ }^{13}$ However, these methods failed to prevent the defects on graphene sheets, which can severely change the intrinsic physic properties of graphene, such as high electrical conductivity and elasticity, and affect the performance of the resultant graphene-based materials. ${ }^{14}$
Thermal exfoliation of GO is believed to be a promising approach for the mass production of graphene materials. ${ }^{15}$ Through this convenient method, the exfoliation and reduction can be achieved simultaneously without the introduction of impurities. The most important factor for thermal exfoliation is the temperature. ${ }^{16} \mathrm{~A}$ high temperature requires more energy consumption and causes more defects on graphene sheets than at low temperature. Moreover, higher temperature also causes more topological defects due to violent decomposition of functional groups. ${ }^{17}$ Therefore, a mild condition for thermal exfoliation will be of great importance to eliminate the disadvantages caused by the high temperature.

Based on the above, we developed a new material called graphite-reduced graphite oxide (rGO) core-sheath fiber, which was produced by directly using carbon fiber as a raw material. The new material shows a 3D-structured rGO sheet (GS) sheath with a graphite core architecture. The 3Dstructured GS sheath is favorable for ion diffusion due to its interconnected porous system. The graphite core still

Received: March 27, 2014

Accepted: May 12, 2014

Published: May 12, 2014 
maintained its stacked graphitic structure, which enables fast electron transport. Different from approaches producing graphene-based fiber using aqueous GO suspensions as raw materials, ${ }^{11}$ we fabricated the G@3D-GS fiber from graphitic carbon fiber, the outer layer of which was chemically converted to 3D-structured rGO sheets. We denote the fiber as G@3DGS. This paper describes the process of the production and studies the properties of this $3 \mathrm{D}$ structure fiber and the supercapacitor performances of this material.

\section{EXPERIMENTAL SECTION}

2.1. Preparation of Graphite@Graphite Oxide Core-Sheath Fiber. Carbon paper (Toray TGP-H-60 and $190 \mu \mathrm{m}$ thick), based on graphitic carbon fibers, was employed to synthesize G@GO fiber. A piece of carbon paper $(25 \times 40 \mathrm{~mm})$ was put into a mixture of concentrated $\mathrm{H}_{2} \mathrm{SO}_{4}(12 \mathrm{~mL}), \mathrm{K}_{2} \mathrm{~S}_{2} \mathrm{O}_{8}(2.5 \mathrm{~g})$, and $\mathrm{P}_{2} \mathrm{O}_{5}(2.5 \mathrm{~g})$, which was kept at $80^{\circ} \mathrm{C}$ for several hours using a water bath. After the above preoxidation, the carbon paper was then taken out of the solution carefully and washed with deionized water until the rinsewater $\mathrm{pH}$ became neutral. This preoxidized carbon paper was then subjected to oxidation described as follows. $\mathrm{KMnO}_{4}(10 \mathrm{~g})$ was gradually added into concentrated $\mathrm{H}_{2} \mathrm{SO}_{4}(80 \mathrm{~mL})$, which was kept and stirred in an ice-bath. After the potassium permanganate was dissolved completely, the preoxidized carbon paper was put into the mixture. The mixture was then moved into a water bath and stirred at $35{ }^{\circ} \mathrm{C}$ for several hours. Subsequently, the brownish-gray oxidized carbon paper, containing concentrated sulfuric acid, was taken out of the mixture. This oxidized carbon paper was placed in water vapor at $50{ }^{\circ} \mathrm{C}$ for $\sim 4$ $\mathrm{h}$ to dilute the concentrated sulfuric acid. After the vapor treatment, the product was washed with deionized water to remove residual sulfuric acid and soluble salt, followed by $1 \%$ hydrogen peroxide to reduce the residual permanganate and manganese dioxide to soluble manganese sulfate. Finally, the product was dried at $60{ }^{\circ} \mathrm{C}$ for $24 \mathrm{~h}$. After the above treatment, the outer layer of the carbon fiber was oxidized to GO with the inner core remaining as graphite. We denote this core-sheath structure fiber as G@GO.

2.2. Exfoliation and Reduction of G@GO. Oxidized carbon paper, prepared as described above, consisted of G@GO fibers. Oxygen-containing groups make the atomic-thick layers hydrophilic. As a result, these oxidized layers can be exfoliated in water under ultrasonication, causing breakage of the G@GO fibers. To obtain undamaged fiber, the G@GO fiber was thermally exfoliated. The dried G@GO was thermally exfoliated in a simple horizontal quartz tube. After being flushed with nitrogen for $15 \mathrm{~min}$, the sample was inserted into a tube furnace preheated to $300{ }^{\circ} \mathrm{C}$. This process removes a large portion of the oxygen groups attached to the sheets and exfoliates the GO layer into 3D rGO sheets. ${ }^{18}$ Afterward, the furnace was heated to $600{ }^{\circ} \mathrm{C}$ at a heating rate of $5^{\circ} \mathrm{C} \mathrm{min}{ }^{-1}$ and then dwelled at $600{ }^{\circ} \mathrm{C}$ for $30 \mathrm{~min}$ for further deoxidation. The final product was designated as G@3D-GS. $\mathrm{N}_{2}$ gas flowed at $120 \mathrm{~cm}^{3} \mathrm{~min}^{-1}$ during the full duration of the thermal treatment.

2.3. Sample Characterization. X-ray powder diffraction (XRD) patterns were obtained with an X'Pert PRO Powder diffractometer (PANalytical Co., The Netherlands), using Ni-filtered $\mathrm{Cu} \mathrm{K} \alpha$ radiation scanned from 5 to $70^{\circ}$ (in $2 \theta$ ). Scanning electron microscopy (SEM) was carried out in a JSM 6301F instrument (JEOL, Japan). Highresolution transmission electron microscopy (HRTEM) was carried out in a JEM-2100F instrument (JEOL, Japan). X-ray photoelectron spectroscopy (XPS) analysis was performed with a Kratos AXIS Ultra X-ray photoelectron spectrometer. A Confocal Raman Microscope (inVia-Reflex, Renishaw, England) was used to obtain Raman spectra and a linearly polarized $\mathrm{He}-\mathrm{Ne}$ laser Spectra Physics model 127, $(\lambda=$ $532 \mathrm{~nm}$, nominally $60 \mathrm{~mW}$ output) was used as the excitation source. Thermogravimetric analysis (TGA) and differential scanning calorimetry (DSC) analysis were carried out in a TGA/DSC 1/1600 thermogravimetric analyzer (Mettler Toledo, Switzerland), in which G@GO was studied under $\mathrm{N}_{2}$ atmosphere with a heating rate of $1{ }^{\circ} \mathrm{C}$ $\mathrm{min}^{-1}$ from 30 to $800{ }^{\circ} \mathrm{C}$. Surface area and pore size characterization analysis was performed by nitrogen $(77 \mathrm{~K})$ adsorption/desorption experiments with an ASAP 2020 instrument (Micromeritics, USA).

2.4. Electrochemical Measurement. The electrochemical properties of the samples were characterized using a two-electrode system with a CHI660C electrochemical workstation at room temperature. The freestanding paper-like G@3D-GS sample was directly used as the electrodes. Free-standing G@3D-GS fiber paper was used as the working electrodes directly, with $6 \mathrm{M} \mathrm{KOH}$ as the electrolyte, and TF4035 (NKK, Japan) as the separator (see Figure S1, Supporting Information). Each working electrode contained $5 \mathrm{mg}$ of G@3D-GS fiber paper with a coating area of $1 \mathrm{~cm}^{2}$. The cyclic voltammetry $(\mathrm{CV})$ and galvanostatic charge/discharge (GC) measurements were carried out in a voltage range between 0 and $0.9 \mathrm{~V}$. The electrochemical impedance spectroscopy (EIS) measurements were performed in a frequency range from $100 \mathrm{kHz}$ to $10 \mathrm{mHz}$ with an $\mathrm{AC}$ amplitude of $5 \mathrm{mV}$.

\section{RESULTS AND DISCUSSION}

As we have described above, the G@3D-GS fiber was produced in three steps: preoxidation of carbon fiber with persulfate, followed by oxidation with permanganate and thermal exfoliation. Graphite@graphite oxide (G@GO) fiber was obtained after the first two steps. The thermal exfoliation and reduction of G@GO gave the final G@3D-GS (Figure 1). (a) Carbon fiber

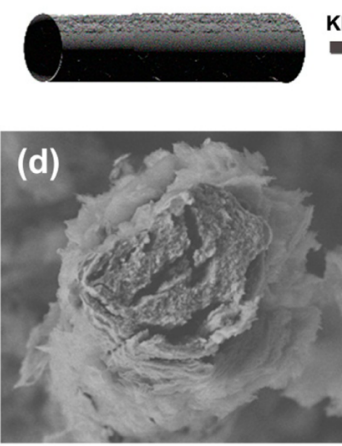

(b) G@GO
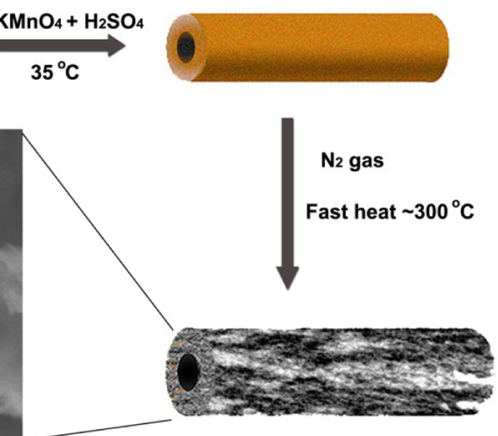

(c) G@3D-GS
Figure 1. Schematic illustration of the synthesis of G@3D-GS: (a) graphitic carbon fiber; (b) G@GO obtained by chemical oxidation of carbon fiber; (c) G@3D-GS, obtained by thermal exfoliation and reduction of G@GO; (d) SEM image of G@3D-GS.

Thermal exfoliation of GO can create defects in the form of holes and vacancies within the basal plane of rGO sheets. Highresolution SEM images of the as-produced G@3D-GS fiber are shown in Figure 2a,b. Compared with previous work showing a worm-like structure, ${ }^{19}$ a fluffy appearance, highly porous and three-dimensional microstructure was readily observed for G@ 3D-GS. The SEM images show this core-sheath structure, with a fluffy rGO sheath and graphite core. After dispersing G@3DGS in solvent using ultrasonication, the fluffy rGO sheath was exfoliated from the fiber and transformed into a wrinkled paperlike structure (Figure 2c). As shown in Figure 2d,e, a large amount of lattice defects are homogeneously distributed throughout the rGO sheets. A previous study reported that rGO sheets generated by thermal exfoliation of GO at moderate temperature tend to overlay with each other, forming aggregated structures with large pores. ${ }^{20}$ The selected area electron diffraction pattern (Figure 2f) shows clear electron diffraction rings, indicating that the honeycomb-like network of carbon atoms partially remained although a certain amount of 


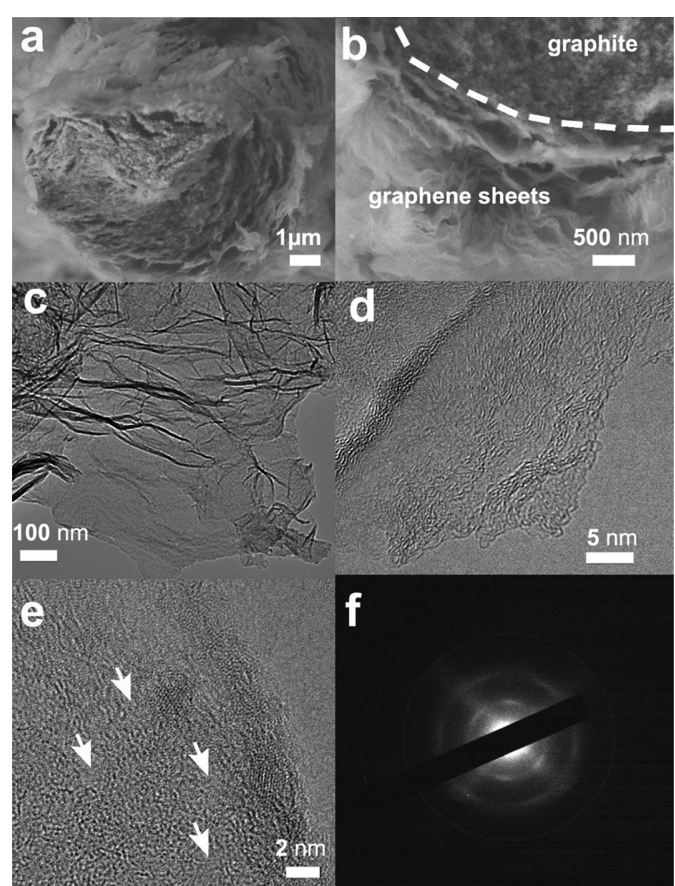

Figure 2. SEM and HRTEM images of G@3D-GS. (a) Cross-section view of G@3D-GS fiber. (b) Detail of graphite core and rGO sheet sheath. (c-e) rGO sheets exfoliated from G@3D-GS fiber. (f) Selected area electron diffraction of rGO sheets.

defects were generated during thermal exfoliation process. The interconnected 3D microstructure of the rGO sheath contributed by the lattice defects and large pores would favor ion diffusion when the G@3D-GS fiber is used as the electrodes of a supercapacitor.

The Raman spectrum of pristine carbon fiber shows three prominent bands of graphitic structures, the D band at 1355 $\mathrm{cm}^{-1}, \mathrm{G}$ band at $1581 \mathrm{~cm}^{-1}$, and $2 \mathrm{D}$ band at $2702 \mathrm{~cm}^{-1}$ (Figure 3). After oxidation, the positions of the $G$ band and the $2 D$ band shift to 1596 and $2685 \mathrm{~cm}^{-1}$, respectively. The change of band positions indicates the destruction of the graphitic structure. $^{21}$ The relative intensity of the $\mathrm{D}$ band is significantly increased upon oxidation of graphite on the carbon fibers, as graphite oxides contain a certain fraction of $\mathrm{sp}^{3}$ carbons. The intensity of the $2 \mathrm{D}$ band decreases after oxidation, which is mainly attributed to the disorder along the $c$-axis, ${ }^{22}$ and is proportional to the change in crystalline graphite crystallite size. $^{23}$

The intensity ratio $I_{\mathrm{D}} / I_{\mathrm{G}}$ (Table 1 ) clearly increases after oxidation, from 0.09 to 0.85 , indicating the defects in the graphitic structure and the formation of oxygen-containing functionalities on the carbon basal plane. ${ }^{24}$ Furthermore, the ratio $I_{\mathrm{D}} / I_{\mathrm{G}}$ of $\mathrm{G} @ 3 \mathrm{D}-\mathrm{GS}$ increased to 1.34 after exfoliation. This change suggests that G@GO to G@3D-GS transformation leaves behind lattice defects and vacancies, and can also be explained by GO layers on the carbon fibers transforming into small size $\mathrm{sp}^{2}$ domains after exfoliation. ${ }^{25}$

The full width at half-maximum (fwhm) of bands depends on the disorder. ${ }^{26}$ Thus, combining with the $I_{\mathrm{D}} / I_{\mathrm{G}}$ ratio, going from carbon fiber to G@GO, the increase of both the fwhm (D) and fwhm (G) is mainly attributed to the increasing disorder after oxidation (Table 1). However, from G@GO to G@3D-GS, the increase of $I_{\mathrm{D}} / I_{\mathrm{G}}$ ratio and the decrease of fwhm show the increasing number of defects and the decrease of disorder after the thermal treatment. The defects were perhaps due to the formation of various polygons by $\mathrm{sp}^{2}$ carbon atoms during the thermal treatment. ${ }^{27}$ The existence of lattice defects on rGO sheets can act as nanochannels between large pores, which enable the rGO sheath to form an interconnected 3D microstructure.

X-ray photoelectron spectroscopy was employed to study the transformation of carbon fiber (Figure 4). The $\mathrm{C} 1 \mathrm{~s}$ spectrum of G@GO and G@3D-GS is shown in Figure 4c,d. The peaks ranging from 286 to $289 \mathrm{eV}$ are typically assigned to oxygencontaining functionalities such as epoxide, hydroxyl, and carboxyl groups. $^{28,29}$ The XPS results suggest that the O/C ratio in G@3D-GS decreased remarkably after the above thermal treatment (oxygen content from 29.1\% to $8.7 \%$ calculated from XPS results), and most of the epoxide and carboxyl functional groups were finally removed (Figure 4d), leaving a larger amount of $\mathrm{sp}^{2}$-bonded carbon. Furthermore, a $\pi$ $\rightarrow \pi^{*}$ shakeup satellite around $291 \mathrm{eV}$, which was mainly contributed by aromatic or conjugated structures, was observed in the C 1s spectrum of G@3D-GS. These results strongly indicated that the GO was reduced. From the results of both Raman spectra and XPS, it can be deduced that the exfoliation and reduction of G@GO results in the re-establishment of conjugated carbon atoms, to form a variety of different structures, not only hexagons. ${ }^{27}$

To investigate the structure of G@GO, we performed X-ray diffraction analysis (Figure 5). Pristine carbon fibers showed a strong (002) peak at $\sim 26.4^{\circ}$, corresponding to highly crystalline graphite with $d$ spacing of $0.337 \mathrm{~nm}$. After oxidation, two diffraction peaks at $\sim 11.8$ and $\sim 26.4^{\circ}$ were observed. The (001) peak at $\sim 11.8^{\circ}$ corresponds to graphite oxide with $d$ spacing of $0.75 \mathrm{~nm}$. Interestingly, a (001) peak split was observed for the least oxidized carbon paper, with two peaks at $\sim 11.8$ and $\sim 12.5^{\circ}$. The splitting of the (001) peak in the XRD pattern can be attributed to the fact that the carbon-carbon bonds go through an intermediate state when the carbon hybridization changes from $\mathrm{sp}^{2}$ to $\mathrm{sp}^{3}$ during the oxidation process. ${ }^{30}$ With the increasing level of oxidation, G@GO shows a predominant peak at $\sim 11.8^{\circ}$, and the (002) peak becomes broader and shifts toward smaller angles (from $\sim 26.4$ to $\sim 24.2^{\circ}$ ), indicating the destruction of the stacked graphitic structure. It is noted that the native graphite $(002)$ peak persists for various oxidation levels, indicating the incomplete oxidation of the carbon fiber. By analyzing the oxidation mechanism, we can conclude that the graphite core of G@GO fiber is composed of this incompletely oxidized graphite. Thermal treatment is an effective technique for exfoliating stacked graphite oxide sheets. ${ }^{20,31}$ After thermal treatment, the (001) peak at $\sim 11.8^{\circ}$ disappears, while the intensity of the $(002)$ peak at $\sim 25.6^{\circ}$ is significantly increased. Compared with pristine carbon fibers, the peak at $\sim 25.6^{\circ}$ is broad and weak, presumably because of the moderate exfoliation leading up to stacked rGO layers.

Graphite oxide on G@GO fiber possesses oxygen-containing functionalities such as carbonyls, carboxyls, and hydroxyls. ${ }^{32}$ These oxygen-containing functionalities expand the interlayer distance and make the oxidized layers hydrophilic. As a result, these layers can be exfoliated into single (or few)-layer graphite oxide through mild sonication in water, causing the damage of the core-sheath structure. Therefore, sonication is considered to be inapplicable to the exfoliation of G@GO. Rapid heating of the graphite oxide makes the oxygen-containing functionalities decompose and spontaneously generates a tremendous 

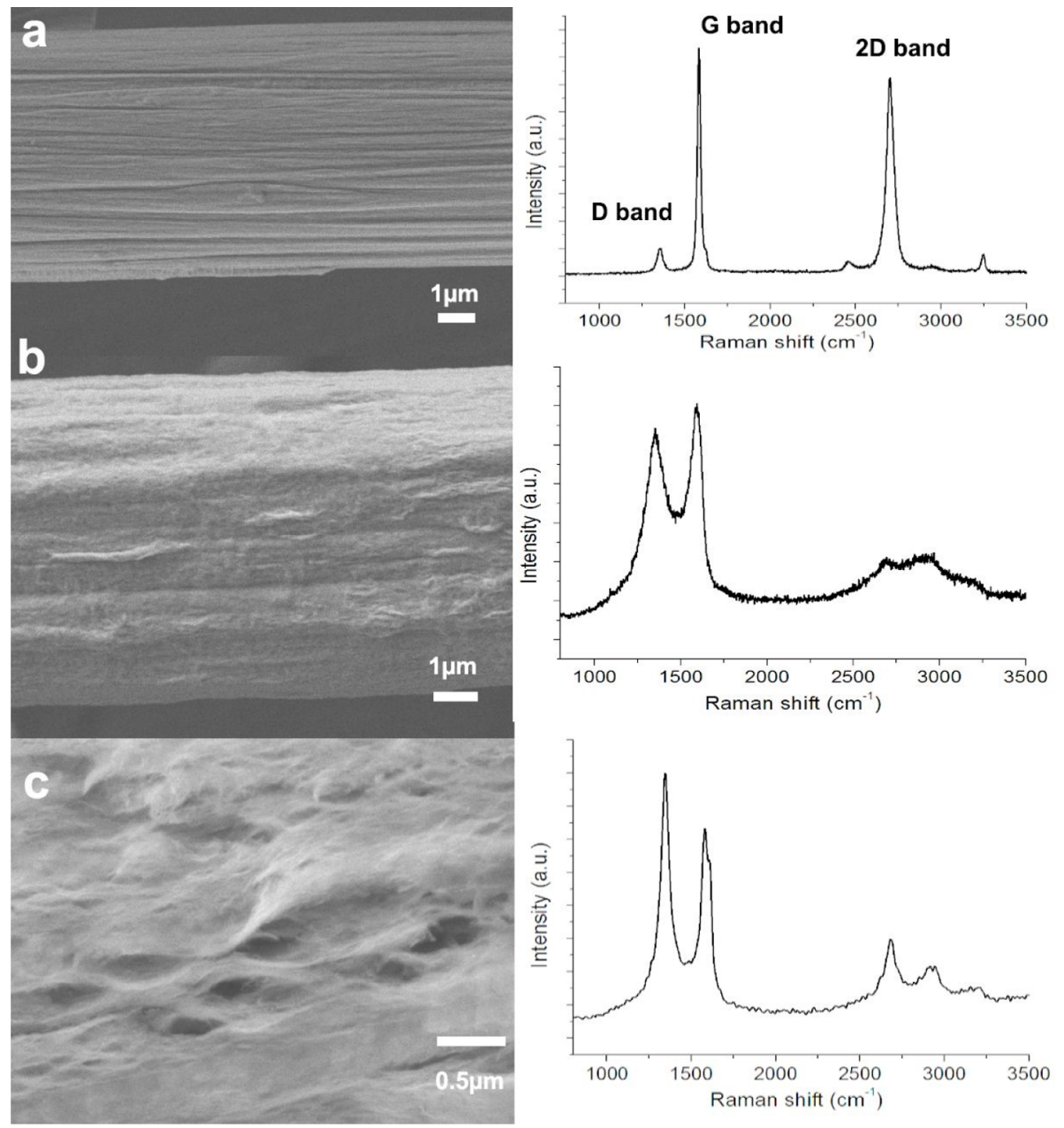

Figure 3. Transformation of carbon fiber to G@3D-GS shown by SEM and Raman spectra. SEM images of the surface of carbon fiber (a), G@GO (b) and G@3D-GS (c). Raman spectra were taken on the surface of fibers shown in SEM images.

Table 1. Comparison of Raman Band Positions, fwhm and $I_{\mathrm{D}} / I_{\mathrm{G}}$ Ratio

\begin{tabular}{cccccc} 
sample & $\begin{array}{c}\mathrm{D} \text { band } \\
\text { position } \\
\left(\mathrm{cm}^{-1}\right)\end{array}$ & $\begin{array}{c}\mathrm{G} \text { band } \\
\text { position } \\
\left(\mathrm{cm}^{-1}\right)\end{array}$ & $\begin{array}{c}\mathrm{D} \text { band } \\
\text { fwhm } \\
\left(\mathrm{cm}^{-1}\right)\end{array}$ & $\begin{array}{c}\mathrm{G} \text { band } \\
\text { fwhm } \\
\left(\mathrm{cm}^{-1}\right)\end{array}$ & $I_{\mathrm{D}} / I_{\mathrm{G}}$ \\
$\begin{array}{c}\text { carbon } \\
\text { fiber }\end{array}$ & 1355 & 1581 & 32 & 22 & 0.09 \\
G@GO & 1354 & 1596 & 179 & 105 & 0.85 \\
G@3D- & 1332 & 1574 & 63 & 46 & 1.34 \\
GS & & & & & \\
\hline
\end{tabular}

amount of gaseous species, which create enough pressure between the oxidized layers to separate the stacked graphite oxide sheets, resulting in an increase in structural defects and yielding a highly porous material. ${ }^{33}$ Such a thermal treatment not only exfoliated but also reduced these GO layers. The dual effects enable the thermal treatment to be very suitable for the exfoliation of G@GO.

TGA/DSC experiments were performed to determine the critical temperature for G@GO exfoliation (Figure S2, Supporting Information). The sharp weight loss at approximately $200{ }^{\circ} \mathrm{C}$ is commonly associated with thermal decomposition of GO layers. However, we found that around this critical temperature $\left(\sim 200{ }^{\circ} \mathrm{C}\right)$, no core-sheath structure was formed. This was probably due to the fact that the pressure generated was not high enough, leading an insufficient reaction.
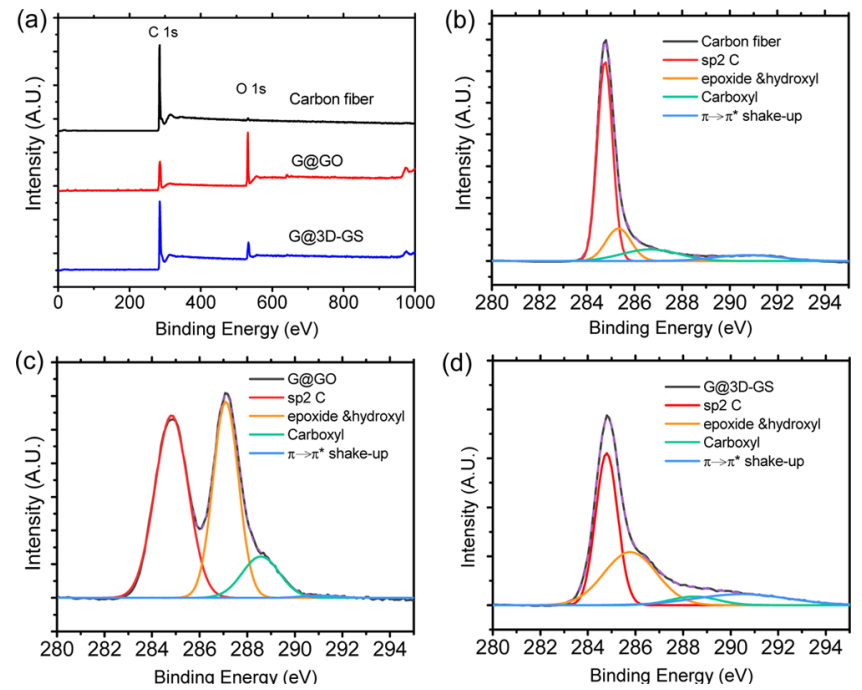

Figure 4. Transformation of carbon fibers shown by XPS analysis. (a) XPS spectra of carbon fiber, G@GO, and G@3D-GS. (b-d) C 1s peak of carbon fiber, G@GO, and G@3D-GS.

However, an excessively violent reaction results in peeling off rGO from the fiber. By varying the exfoliation temperatures from 200 to $600{ }^{\circ} \mathrm{C}$, we finally found that the appropriate temperature was $\sim 300{ }^{\circ} \mathrm{C}$ (Figure S3, Supporting Informa- 


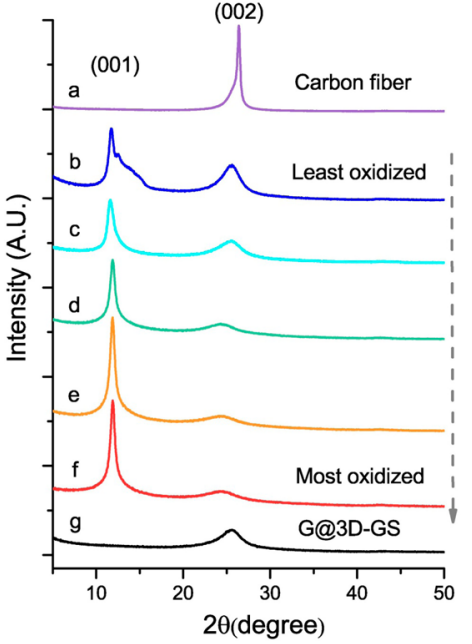

Figure 5. Transformation of carbon fiber to G@3D-GS shown by Xray diffraction analysis. (a) Pristine carbon fibers; (b-f) G@GO obtained at different oxidation levels; (g) G@3D-GS, by thermal exfoliation of G@GO at $300{ }^{\circ} \mathrm{C}$.

tion). Furthermore, G@GO exfoliated at $300{ }^{\circ} \mathrm{C}$ possess a lower $I_{\mathrm{D}} / I_{\mathrm{G}}$ ratio than at $600{ }^{\circ} \mathrm{C}$ (Figure S4, Supporting Information), which implies that lower temperature $\left(300{ }^{\circ} \mathrm{C}\right)$ can cause less defects than higher temperature $\left(600{ }^{\circ} \mathrm{C}\right)$. For the above reasons, a moderate temperature of $300{ }^{\circ} \mathrm{C}$ was used to properly exfoliate these $\mathrm{GO}$, followed by $600{ }^{\circ} \mathrm{C}$ for further deoxidation.

The above results show that the oxidation reaction appears to occur on the surface of the carbon fiber. Therefore, GO layers could be produced on the surface of the carbon fibers. Incomplete oxidation of graphite particles results in the formation of graphite/GO core-shell particles, ${ }^{34}$ thus preoxidation and oxidation of carbon fiber could influence the formation of GO layers. We find that the moderate preoxidation/oxidation results in partial oxidation of carbon fibers, to yield the G@GO core-sheath structure.

Surface and pore size characterization of G@3D-GS was performed by a nitrogen $(77 \mathrm{~K})$ adsorption/desorption experiment (Figure 6). G@3D-GS has a surface area of 255 $\mathrm{m}^{2} \mathrm{~g}^{-1}$. The weak adsorption at relative pressure of $P / P_{0}<0.1$ indicates that no micropores exist in the sample (Figure 6a). The $\mathrm{N}_{2}$ isotherm shows type $\mathrm{H} 3$ hysteresis, which often is associated with slit-shaped pores observed from plate-like materials. ${ }^{35}$ The type II isotherm implies that slit-shaped mesoor macropores exist in the rGO sheets. Strong nitrogen adsorption at $\mathrm{P} / \mathrm{P}_{0}>0.9$ reveals the large open-pore structure of aggregated rGO caused by thermal exfoliation, which can be clearly seen in SEM observation (Figure 2a,b). Pore size distribution analysis was based on density functional theory (DFT). ${ }^{36}$ Figure $6 \mathrm{~b}$ displays the result of pore size distribution analysis using the NLDFT model, assuming a slit/cylindrical pore according to the characteristics of the graphene-based material and the $\mathrm{N}_{2}$ isotherm. The majority of the pore sizes are narrowly distributed at $5.5 \mathrm{~nm}$. The larger pore size distribution ranging from $\sim 8$ to $\sim 30 \mathrm{~nm}$ may be attributed to the violent release of gas, which enables rGO sheets to form a large openpore system.

3D Graphene-based frameworks exhibit interconnected porous architectures, low mass density, and large surface area, which are suitable in many applications, such as Li-ion batteries, capacitive deionization, and supercapacitor electrodes. ${ }^{37}$ However, the defects and vacancies caused by chemical oxidation and thermal exfoliation greatly affect the electrical conductivity of the resultant graphene-based materials. In comparison with pure rGO sheets, G@3D-GS possesses more regular pore structures and conductive paths. The graphite core of G@3DGS fiber can preserve its high conductivity and also bridge the defect rGO sheet sheath for electron transfer, facilitating transport of electrons to the outer region of the fiber. The interconnected 3D microstructure of the rGO sheath contributed by the lattice defects and large pores would favor ion diffusion when the G@3D-GS fiber is used as the electrodes of a supercapacitor. More significantly, there is no need for extra binders or conductive additives, which can greatly simplify the electrode-manufacturing process and enable large-scale applications. The G@3D-GS fiber is expected to provide fast ion diffusion and charge transfer, which are desirable for many applications.

Figure $7 \mathrm{a}$ shows the $\mathrm{CV}$ curves collected from 0 to $0.9 \mathrm{~V}$ with different scan rates ranging from 20 to $100 \mathrm{mV} \mathrm{s}^{-1}$. No obvious distortion was observed as the scan rate increased, indicating a
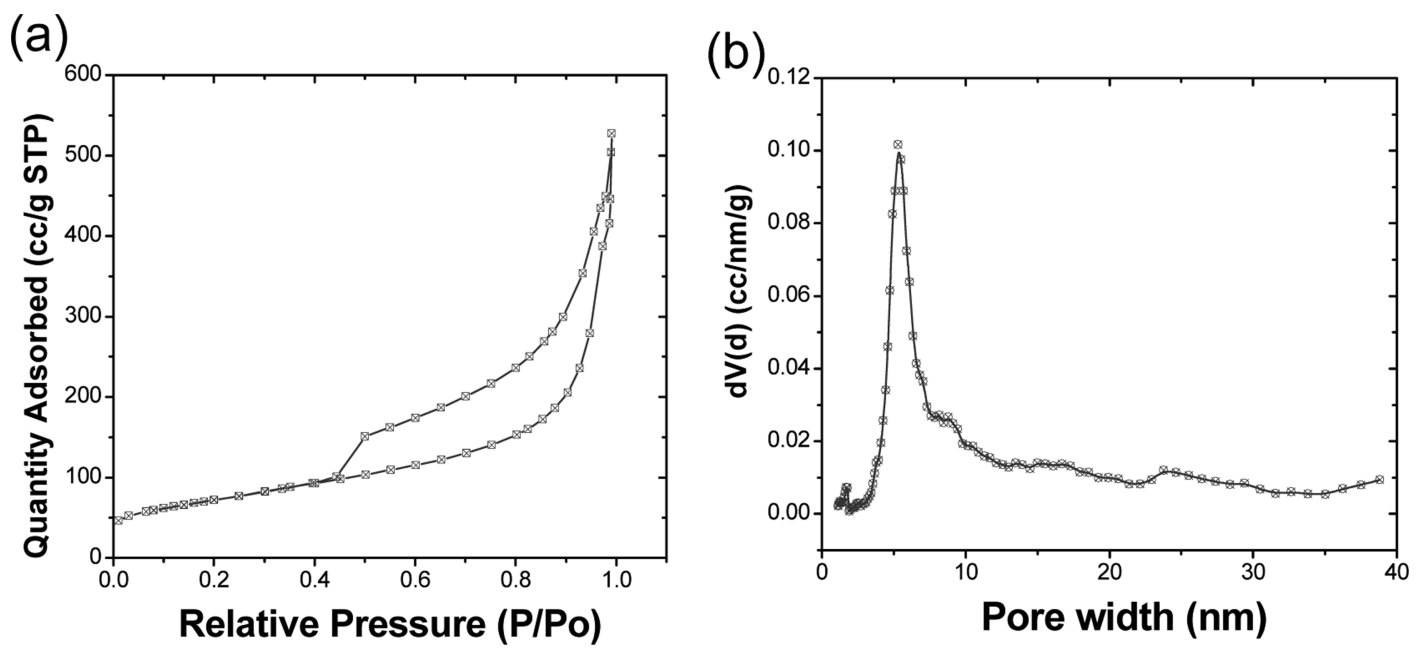

Figure 6. $\mathrm{N}_{2}$ adsorption/desorption analysis of G@3D-GS. (a) $\mathrm{N}_{2}(77 \mathrm{~K})$ isotherms. (b) Pore size distribution of $\mathrm{N}_{2}$ calculated using slit/cylinder NLDFT model. 

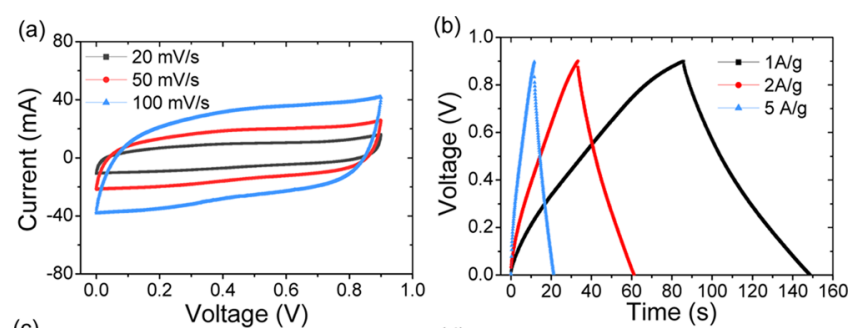

(c)
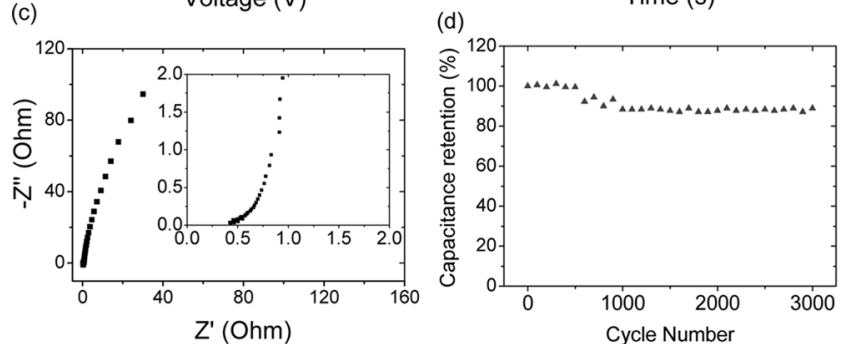

Figure 7. Supercapacitor performance of G@3D-GS in $6 \mathrm{M} \mathrm{KOH}$ aqueous solution. (a) $\mathrm{CV}$ curves for different scan rates with the potential range from 0 to $0.9 \mathrm{~V}$. (b) Galvanostatic charge/discharge curves of G@3D-GS electrodes under different current densities. (c) Nyquist plot of G@3D-GS. Inset shows the high-frequency area. (d) Cycling stability of G@3D-GS at a current density of $1 \mathrm{~A} \mathrm{~g}^{-1}$.

highly reversible characteristic within the applied potential range. The $\mathrm{CV}$ curves are near rectangular in shape without obvious Faradaic current at the scan rate of $100 \mathrm{mV} \mathrm{s}^{-1}$, reflecting the characteristic of electric double layer capacitance with an excellent capacitive behavior and low internal resistance. This performance can be attributed to the combination of 3D frameworks for accessibility of the electrolyte ions, and conductive paths based on the graphitic core structure. The triangular shape of the galvanostatic charge/ discharge plots indicated good charge transfer behavior of ions in the G@3D-GS electrode and ideal capacitor behavior. The specific capacitance was calculated from the discharge curve using the formula $C s=I /(\mathrm{m}(\Delta V / \Delta t)$. Based on the above formula, the specific capacitances of G@3D-GS paper were 140, 124, and $108 \mathrm{~F} \mathrm{~g}^{-1}$ obtained at current densities of 1,2 , and $5 \mathrm{~A} \mathrm{~g}^{-1}$, respectively (Figure S5, Supporting Information).

Figure 7c shows the Nyquist plot of the G@3D-GS paper electrode. The imaginary part sharply increases at low frequency and a nearly vertical line is observed, indicating a pure capacitive behavior for G@3D-GS. The Warburg curve in Figure 7c (inset) is quite short, indicating good accessibility for electrolyte ion transfer to the rGO sheet surface. The low equivalent series resistance $\left(R_{\mathrm{ESR}}\right)$ value of $0.45 \mathrm{ohm}$ and small loop at high frequency indicate an extremely small resistance between the graphite core and rGO sheet sheath. This is mainly because the rGO sheets are grown rather than coated on the graphite core. The small resistance is also confirmed by observing the negligible internal resistance drops of the discharge curves in Figure $7 \mathrm{~b}$. The power density, calculated from the discharge curve using $P=V^{2} / 4 M R_{\mathrm{ESR}}$ was $45 \mathrm{~kW} / \mathrm{kg}$ ( $R_{\mathrm{ESR}}$ is equivalent series resistance; $M$ is the total weight of the two carbon electrodes). The galvanostatic charge/discharge cycling of G@3D-GS was performed to study the long-term stability of this material. After 3000 cycles performed at a current density of $1 \mathrm{~A} \mathrm{~g}^{-1}$, there is a $10 \%$ loss of capacity compared with the starting value, indicating the good cycling stability of G@3D-GS (Figure 7d).

The excellent electrochemical performance of G@3D-GS makes it possible to engineer supercapacitor electrodes to achieve a high specific capacitance, high power density, and especially low cost. The 3D rGO sheets sheath with high surface area and regular pore structure facilitate ion diffusion between the electrolyte and electrodes. The surface area normalized capacitance reached as high as $59.4 \mu \mathrm{F} \mathrm{cm}$, indicating the effective use of surface area (Table S1, Supporting Information). ${ }^{38}$ Importantly, unlike other graphene-based materials without such an excellent electron transfer path, the graphite core of G@3D-GS fiber offers an "electric cable" that enables fast electron transfer. This G@3DGS material can be easily produced from carbon fiber, which could be obtained at low cost. The processes used to synthesize this material can easily be put into practice. For supercapacitor manufacturing, we propose that the convenient method presented will provide an efficient process and open the possibility to produce high performance supercapacitor materials at low cost for future energy storage applications.

\section{CONCLUSIONS}

We report an economic and easy method to synthesize graphite-rGO core-sheath fiber. This G@3D-GS fiber was manufactured by moderate oxidation of graphitic carbon fiber to form G@GO, followed by critical thermal exfoliation. The G@3D-GS fiber with a unique 3D framework rGO sheath offers an ideal pore structure and large surface area. The graphite core preserves the high conductivity of carbon fiber, which enables fast electron transfer between the 3D rGO sheath and the electrolyte. We propose that this work will benefit the development of graphene-based materials and various advanced energy electrochemical storage devices.

\section{ASSOCIATED CONTENT}

\section{Supporting Information}

Schematic of the two-electrode supercapacitor construction; TGA/DSC analysis of G@GO; SEM images of G@3D-GS obtained at different exfoliation temperatures; comparison of graphite-reduced graphite oxide core-sheath fiber by Raman spectrum at different exfoliation temperatures; specific capacitance of G@3D-GS at different current densities; comparison of typical values for electrochemical double-layer capacitance of carbonaceous materials. This material is available free of charge via the Internet at http://pubs.acs.org.

\section{AUTHOR INFORMATION}

\section{Corresponding Author}

*Jiuhui Qu. Tel: +86-10-62849151. E-mail: jhqu@rcees.ac.cn. Notes

The authors declare no competing financial interest.

\section{ACKNOWLEDGMENTS}

This work was supported by the National Science Fund for Distinguished Young Scholars of China (Grant No. 51225805) and the National Natural Science Foundation of China (Grant No. 51222802).

\section{REFERENCES}

(1) Schwierz, F. Graphene transistors. Nat. Nanotechnol. 2010, 5, 487-496.

(2) Ramanathan, T.; Abdala, A. A.; Stankovich, S.; Dikin, D. A.; Herrera Alonso, M.; Piner, R. D.; Adamson, D. H.; Schniepp, H. C.; ChenX; Ruoff, R. S.; Nguyen, S. T.; Aksay, I. A.; Prud'Homme, R. K.; Brinson, L. C. Functionalized graphene sheets for polymer nanocomposites. Nat. Nanotechnol. 2008, 3, 327-331. 
(3) Kim, H.; Abdala, A. A.; Macosko, C. W. Graphene/Polymer Nanocomposites. Macromolecules 2010, 43, 6515-6530.

(4) Lee, C.; Wei, X.; Kysar, J. W.; Hone, J. Measurement of the Elastic Properties and Intrinsic Strength of Monolayer Graphene. Science 2008, 321, 385-388.

(5) Segal, M. Selling graphene by the ton. Nat. Nanotechnol. 2009, 4, $612-614$.

(6) Xu, Y.; Sheng, K.; Li, C.; Shi, G. Self-Assembled Graphene Hydrogel via a One-Step Hydrothermal Process. ACS Nano 2010, 4, 4324-4330.

(7) Sun, H.; Xu, Z.; Gao, C. Multifunctional, ultra-flyweight, synergistically assembled carbon aerogels. Adv. Mater. 2013, 25, 2554-60.

(8) Hu, H.; Zhao, Z.; Wan, W.; Gogotsi, Y.; Qiu, J. Ultralight and Highly Compressible Graphene Aerogels. Adv. Mater. 2013, 25, 22192223.

(9) Cong, H. P.; Ren, X. C.; Wang, P.; Yu, S. H. Wet-spinning assembly of continuous, neat, and macroscopic graphene fibers. Sci. Rep. 2012, 2, 613.

(10) Dong, Z.; Jiang, C.; Cheng, H.; Zhao, Y.; Shi, G.; Jiang, L.; Qu, L. Facile Fabrication of Light, Flexible and Multifunctional Graphene Fibers. Adv. Mater. 2012, 24, 1856-1861.

(11) Meng, Y.; Zhao, Y.; Hu, C.; Cheng, H.; Hu, Y.; Zhang, Z.; Shi, G.; Qu, L. All-graphene core-sheath microfibers for all-solid-state, stretchable fibriform supercapacitors and wearable electronic textiles. Adv. Mater. 2013, 25, 2326-31.

(12) Xu, Z.; Gao, C. Graphene chiral liquid crystals and macroscopic assembled fibres. Nat. Commun. 2011, 2, 571.

(13) Li, C.; Shi, G. Three-dimensional graphene architectures. Nanoscale 2012, 4, 5549-63.

(14) Huang, Y.; Liang, J.; Chen, Y. An Overview of the Applications of Graphene-Based Materials in Supercapacitors. Small 2012, 8, 18051834.

(15) Sun, G.; Zheng, L.; Zhan, Z.; Zhou, J.; Liu, X.; Li, L. Actuation triggered exfoliation of graphene oxide at low temperature for electrochemical capacitor applications. Carbon 2014, 68, 748-754.

(16) Zhang, C.; Lv, W.; Xie, X.; Tang, D.; Liu, C.; Yang, Q.-H. Towards low temperature thermal exfoliation of graphite oxide for graphene production. Carbon 2013, 62, 11-24.

(17) Schniepp, H. C.; Li, J.-L.; McAllister, M. J.; Sai, H.; HerreraAlonso, M.; Adamson, D. H.; Prud'homme, R. K.; Car, R.; Saville, D. A.; Aksay, I. A. Functionalized Single Graphene Sheets Derived from Splitting Graphite Oxide. J. Phys. Chem. B 2006, 110, 8535-8539.

(18) Botas, C.; Álvarez, P.; Blanco, C.; Santamaría, R.; Granda, M.; Gutiérrez, M. D.; Rodríguez-Reinoso, F.; Menéndez, R. Critical temperatures in the synthesis of graphene-like materials by thermal exfoliation-reduction of graphite oxide. Carbon 2013, 52, 476-485.

(19) Celzard, A.; Marêché, J. F.; Furdin, G. Modelling of exfoliated graphite. Prog. Mater. Sci. 2005, 50, 93-179.

(20) Lv, W.; Tang, D.-M.; He, Y.-B.; You, C.-H.; Shi, Z.-Q.; Chen, X.C.; Chen, C.-M.; Hou, P.-X.; Liu, C.; Yang, Q.-H. Low-Temperature Exfoliated Graphenes: Vacuum-Promoted Exfoliation and Electrochemical Energy Storage. ACS Nano 2009, 3, 3730-3736.

(21) Kudin, K. N.; Ozbas, B.; Schniepp, H. C.; Prud'homme, R. K.; Aksay, I. A.; Car, R. Raman Spectra of Graphite Oxide and Functionalized Graphene Sheets. Nano Lett. 2007, 8, 36-41.

(22) Ferrari, A. C.; Robertson, J. Resonant Raman spectroscopy of disordered, amorphous, and diamondlike carbon. Phys. Rev. B 2001, 64, 075414.

(23) Bokova, S. N.; Obraztsova, E. D.; Grebenyukov, V. V.; Elumeeva, K. V.; Ishchenko, A. V.; Kuznetsov, V. L. Raman diagnostics of multi-wall carbon nanotubes with a small wall number. Phys. Status Solidi B 2010, 247, 2827-2830.

(24) Ferrari, A. C.; Meyer, J. C.; Scardaci, V.; Casiraghi, C.; Lazzeri, M.; Mauri, F.; Piscanec, S.; Jiang, D.; Novoselov, K. S.; Roth, S.; Geim, A. K. Raman Spectrum of Graphene and Graphene Layers. Phys. Rev. Lett. 2006, 97, 187401.

(25) Tuinstra, F.; Koenig, J. L. Raman Spectrum of Graphite. J. Chem. Phys. 1970, 53, 1126-1130.
(26) Ferrari, A. C.; Rodil, S. E.; Robertson, J. Interpretation of infrared and Raman spectra of amorphous carbon nitrides. Phys. Rev. B 2003, 67, 1553061-15530620.

(27) Banhart, F.; Kotakoski, J.; Krasheninnikov, A. V. Structural Defects in Graphene. ACS Nano 2010, 5, 26-41.

(28) Yang, D.; Velamakanni, A.; Bozoklu, G.; Park, S.; Stoller, M.; Piner, R. D.; Stankovich, S.; Jung, I.; Field, D. A.; Ventrice, C. A., Jr; Ruoff, R. S. Chemical analysis of graphene oxide films after heat and chemical treatments by X-ray photoelectron and Micro-Raman spectroscopy. Carbon 2009, 47, 145-152.

(29) Fan, X.; Peng, W.; Li, Y.; Li, X.; Wang, S.; Zhang, G.; Zhang, F. Deoxygenation of Exfoliated Graphite Oxide under Alkaline Conditions: A Green Route to Graphene Preparation. Adv. Mater. 2008, 20, 4490-4493.

(30) Nakajima, T.; Matsuo, Y. Formation process and structure of graphite oxide. Carbon 1994, 32, 469-475.

(31) McAllister, M. J.; Li, J.-L.; Adamson, D. H.; Schniepp, H. C.; Abdala, A. A.; Liu, J.; Herrera-Alonso, M.; Milius, D. L.; Car, R.; Prud'homme, R. K.; Aksay, I. A. Single Sheet Functionalized Graphene by Oxidation and Thermal Expansion of Graphite. Chem. Mater. 2007, $19,4396-4404$

(32) Hummers, W. S.; Offeman, R. E. Preparation of Graphitic Oxide. J. Am. Chem. Soc. 1958, 80, 1339-1339.

(33) Jung, I.; Field, D. A.; Clark, N. J.; Zhu, Y.; Yang, D.; Piner, R. D.; Stankovich, S.; Dikin, D. A.; Geisler, H.; Ventrice, C. A.; Ruoff, R. S. Reduction Kinetics of Graphene Oxide Determined by Electrical Transport Measurements and Temperature Programmed Desorption. J. Phys. Chem. C 2009, 113, 18480-18486.

(34) Kovtyukhova, N. I.; Ollivier, P. J.; Martin, B. R.; Mallouk, T. E.; Chizhik, S. A.; Buzaneva, E. V.; Gorchinskiy, A. D. Layer-by-Layer Assembly of Ultrathin Composite Films from Micron-Sized Graphite Oxide Sheets and Polycations. Chem. Mater. 1999, 11, 771-778.

(35) Sing, K. S. W.; Everett, D. H.; Haul, R. A. W.; Moscou, L.; Pierott, R. A.; Rouquerol, J.; Siemieniewsk, T. Reporting physisorption data for gas solid systems with special reference to the determination of surface area and porosity. Pure Appl. Chem. 1985, 57, 17.

(36) Ravikovitch, P. I.; Vishnyakov, A.; Russo, R; Neimark, A. V. Unified Approach to Pore Size Characterization of Microporous Carbonaceous Materials from $\mathrm{N}_{2}, \mathrm{Ar}$, and $\mathrm{CO}_{2}$ Adsorption Isotherms. Langmuir 2000, 16, 2311-2320.

(37) Zhang, L. L.; Zhao, X.; Stoller, M. D.; Zhu, Y.; Ji, H.; Murali, S.; Wu, Y.; Perales, S.; Clevenger, B.; Ruoff, R. S. Highly Conductive and Porous Activated Reduced Graphene Oxide Films for High-Power Supercapacitors. Nano Lett. 2012, 12, 1806-1812.

(38) Largeot, C.; Portet, C.; Chmiola, J.; Taberna, P.-L.; Gogotsi, Y.; Simon, P. Relation between the Ion Size and Pore Size for an Electric Double-Layer Capacitor. J. Am. Chem. Soc. 2008, 130, 2730-2731. 